\title{
INTENSIFICATION ET OPPOSITION : \\ L'ADJECTIF INTENSIF À VALEUR ARGUMENTATIVE
}

\author{
Véronique LENEPVEU
}

Université de Caen, CRISCO FRE 28 05, CNRS

\section{Introduction}

Nous cherchons à décrire le fonctionnement de l'adjectif intensif au sein du SN et à mettre en évidence son rôle dans l'enchaînement argumentatif en faisant intervenir la dimension axiologique de l'argumentation. Dans cette perspective, nous nous sommes intéressée au fonctionnement d'adjectifs primaires ( $c f$. Goes, 1999 : 45-46), préférentiellement antéposés en fonction d'épithète et susceptibles de prendre une valeur intensive ${ }^{1}$. Nous voulons précisément observer leur comportement lorsqu'ils sont mis en relation avec un nom à valeur appréciative. Pour exemples :

[1] de larges bénéfices, de gros avantages, une maigre consolation, une faible amélioration, un léger retard, de nets progrès, ...

Il s'agit de montrer comment la présence de l'adjectif intensif constitue un facteur susceptible d'influer sur la force évaluative du SN et comment ce facteur se combine avec d'autres facteurs également décisifs pour déterminer la valeur axiologique ${ }^{2}$ du SN.

\section{L'adjectif modificateur « réalisant » ou « déréalisant »}

\footnotetext{
${ }^{1}$ Le lien entre la position antéposée de l'adjectif et une valeur intensive a déjà été montré entre autres par Blinkenberg (1933 : 67-72) : plus le sens de l'adjectif se rapproche de celui de grand/petit ou bon/mauvais, plus l'adjectif a tendance à s'antéposer et à prendre une valeur intensive. Voir aussi Wilmet (1980) et Martin (1986). ${ }^{2}$ Valeur axiologique est pris ici dans le sens de jugement de valeur et non dans le sens restrictif de jugement moral.
} 
1. 1. Pour étudier le fonctionnement argumentatif de ces adjectifs épithètes intensifs ${ }^{3}$, nous admettons les hypothèses de Ducrot (1995) et de Anscombre éd. (1995) selon lesquelles la signification d'un prédicat comporte en elle-même une certaine gradualité, que l'on peut identifier aux différentes forces avec lesquelles s'appliquent les topoï convoqués par ce prédicat et que l'on peut appréhender en observant le fonctionnement de ce que Ducrot (1995) appelle modificateurs « réalisants » ou « déréalisants ». Des adverbes et des adjectifs sont à même de jouer le rôle de modificateur de prédicat, c'est-à-dire à même de renforcer ou au contraire de faire obstacle à l'application des topoï constitutifs d'un prédicat donné ${ }^{4}$. Ainsi, un adverbe de degré peut-il diminuer ou augmenter l'applicabilité du prédicat. Soit les exemples :

[2] Paul est très malade, il a besoin de médicaments.

[3] Paul est un peu malade, il a besoin de médicaments.

[4] Paul est peu malade, il n'a pas besoin de médicaments.

Si très accroît la force avec laquelle on applique le ou les topoï qu'il convoque et fonctionne comme modificateur réalisant, l'adverbe un peu diminue au contraire la force avec laquelle s'appliquent les topoï tout en conservant cependant l'orientation intrinsèque du prédicat : il est modificateur déréalisant atténuateur. Enfin, peu est modificateur déréalisant inverseur en ce sens où il inverse l'orientation argumentative intrinsèque du prédicat. Les modificateurs déréalisants peuvent donc avoir deux fonctions : atténuer tout en conservant l'orientation argumentative du prédicat ou bien inverser cette orientation.

\footnotetext{
${ }^{3}$ Syntaxiquement, il est difficile de circonscrire exactement ce groupe d'adjectifs peu homogène. Le test de la mise en attribut donne des résultats très variables (voir à ce sujet Lenepveu, 2004), la postposition de l'adjectif épithète n'est pas systématiquement exclue, elle peut même être facilitée par la présence d'un adverbe de degré (une amélioration assez faible), seulement la tendance est très nettement à l'antéposition et, sur ce point, nos adjectifs s'apparentent aux adjectifs modaux (Milner, 1967). Sur le plan sémantique, ces adjectifs ne peuvent pas cependant être appréhendés en termes d'appartenance d'un objet à une classe comme le fait Milner, nous les considérons plutôt comme des modalisateurs de désignation nominale au sens de Riegel (2005) en précisant que la modalisation se définit ici en termes d'intensification, l'adjectif indiquant un degré de validation de la dénomination choisie.

${ }^{4}$ Le mot prédicat n'est pas à prendre ici au sens logique du terme, ni au sens grammatical, les prédicats sont les termes introducteurs de topoï sur lesquels peuvent porter les modificateurs.
} 
Un adjectif peut aussi avoir ce rôle de modificateur ( $c f$. Ducrot, 1996 et Romero, 2001) et attester de la gradualité intrinsèque d'un prédicat. Deux exemples de Ducrot (1996: 201) montrent, à cet égard, qu'en position d'épithète antéposée, un adjectif primaire est à même de fonctionner comme inverseur ou comme atténuateur. Ces exemples font référence au référendum sur l'indépendance du Québec qui a eu lieu en octobre 1995, et qui a vu le «non » à l'indépendance l'emporter avec seulement une très légère majorité. Dans le premier énoncé qui est attesté, l'adjectif courte inverse les possibilités argumentatives du mot victoire, une paraphrase serait Parce qu'elle est courte... :

[5] La courte victoire du non ébranle la fédération canadienne. (Le Monde, 1/11/1995)

Dans le second exemple, qui est cette fois-ci fabriqué par l'auteur, l'adjectif courte affaiblit mais ne modifie pas l'orientation intrinsèque de victoire, il est atténuateur. La paraphrase proposée est alors une proposition subordonnée concessive Bien qu'elle soit courte.... Soit cet exemple :

[6] La courte victoire du non laisse ses chances à la fédération canadienne.

1. 2. Sur la base de cette analyse, nous voulons montrer la nécessité de prendre en compte la valeur appréciative du substantif, l'adjectif épithète antéposé de valeur intensive prenant, en contexte, une valeur argumentative qui consiste à renforcer, atténuer ou inverser la valeur appréciative du substantif.

Il est notable en effet qu'un adjectif, indicateur de haut degré et modificateur réalisant, aura un effet appréciatif positif si le substantif est marqué positivement (une forte amélioration, de larges bénéfices) et un effet appréciatif négatif si le substantif est marqué négativement (un gros retard), c'est-à-dire qu'une intensité forte avec une évaluation positive renforce le caractère positif de l'évaluation tandis qu'une intensité forte avec une évaluation négative renforce le caractère négatif de l'évaluation 5 .

\footnotetext{
${ }^{5}$ Nous définissons la dimension axiologique de l'argumentation par la polarité positif/négatif souvent assimilée à l'opposition bon/mauvais (Galatanu, 2001) ou euphorique/dysphorique (Raccah, 2005).
} 
Il est également remarquable que si l'adjectif indique un faible degré et fonctionne comme modificateur déréalisant, il atténue ou inverse le caractère positif ou négatif de l'évaluation. C'est ce que nous avons pu observer, à partir d'exemples sélectionnés sur Google (G) ou repérés dans Le Monde (CD-ROM, 1999-2002), en étudiant, tout d'abord, l'adjectif faible $e^{6}$ susceptible de fonctionner tantôt comme atténuateur, tantôt comme inverseur par rapport à un terme marqué axiologiquement comme amélioration. L'exemple [7] illustre le fonctionnement de faible comme atténuateur :

[7] La vente d'équipement de maison a l'air de reprendre. Si nous avons connu une bonne période estivale, septembre n'est pas à proprement parler un mois de reprise. Nous tablons toutefois sur une faible amélioration de la consommation pour l'année prochaine (Reprise économique ? Coup de sonde dans la région, La Presse, Riviera Chablais, 01/10/97, G)

Faible affaiblit mais ne modifie pas l'orientation axiologique positive de l'énoncé. En revanche, dans les exemples [8] et [9], une faible amélioration confère une orientation axiologique négative à l'énoncé global du fait de l'inversion de la valeur positive, c'est-à-dire intrinsèquement méliorative, de amélioration :

[8] Alors que la plupart des pays développés introduisent de plus en plus fréquemment l'ordinateur dans les salles de classe (...) une association américaine de protection de l'enfance vient de jeter un pavé dans la mare en publiant un rapport qui pointe les effets nocifs de l'ordinateur sur les plus jeunes élèves.

Les auteurs du rapport rappellent en effet que la plupart des études constatent une faible amélioration des résultats aux exercices lorsque l'élève s'entraîne sur un ordinateur. Plus profondément, l'association pointe les effets néfastes que peut avoir la pratique intensive de l'informatique sur les plus jeunes enfants. (L'ordinateur est-il bon pour les enfants? Alliance for Childhood jette le doute, P. Mounier, Homo Numericus, 01/02/02, G)

[9] La Deuxième Conférence des Nations Unies sur les établissements humains (Habitat II) poursuit son débat général.

\footnotetext{
${ }^{6}$ Alors que les travaux sur la gradation se sont centrés, ces dernières années, essentiellement sur les valeurs du haut degré, à la suite des analyses de Kennedy (1999), Kennedy \& McNally (1999) (Voir à ce sujet Hadermann, Pierrard \& Van Raemdonck (éds), 2007), nous étudions ici les valeurs de bas degré.
} 
M. Per Nygaard (Norvège) : Habitat II est un événement crucial. Cette conférence nous donne l'occasion de débattre des objectifs et des principes, des stratégies et des mesures et de parvenir à un accord afin de nous engager en faveur d'un Programme pour l'habitat. Sans une mise en oeuvre efficace de ce programme au cours des mois et des années qui suivront cette conférence, une faible amélioration est à attendre. (Deuxième Conférence des Nations Unies Habitat II, Service des Informations et des Accrédidations, 20/12/02, G)

On voit donc que faible qui, sémantiquement, possède une valeur d'atténuation, va sur le plan pragmatique tantôt affaiblir, tantôt inverser le trait positif associé à amélioration, c'est-à-dire que la valeur positive de amélioration peut, dans certains cas, être suffisamment affaiblie pour pouvoir s'inverser.

Pour rendre compte de ce phénomène d'inversion et décrire l'orientation scalaire du SN, nous posons l'existence d'une échelle unique mais à double orientation, les échelons (dont le nombre est a priori illimité) s'ordonnant selon deux pôles, un pôle positif et un pôle négatif. Sur cette échelle, une faible amélioration pourra désigner un faible degré orienté positivement (soit à droite du repère marqué par I) ou bien un faible degré orienté négativement (soit à gauche du repère $\mathrm{I})^{7}$ :

\footnotetext{
${ }^{7}$ Poser deux échelles distinctes comme le propose Ducrot (1980) ou Rivara (1993) ne permet pas de décrire le mécanisme qui permet de passer d'une orientation à l'autre, d'où la nécessité pour nous de poser une échelle à double orientation. Ce type d'échelle est implicite chez Nøjgaard (1995 : § 797), le système de l'intensification s'ordonnant selon deux pôles (haut degré/bas degré), l'intensification est toujours interprétée comme une augmentation ou comme une diminution par rapport à un degré neutre que présuppose toute opération d'intensification. On trouve aussi cette échelle à double orientation chez Gosselin (2005: 51), mais pour décrire cette fois-ci les modalités sous forme de continuums.
} 


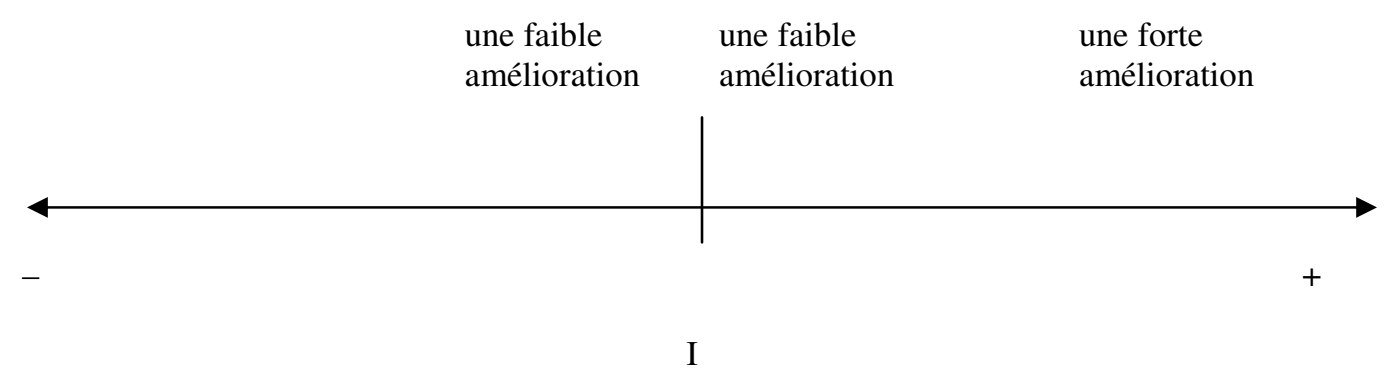

\section{L'adjectif déréalisant : atténuateur ou inverseur ? Quels critères ?}

2. 1. Outre la valeur appréciative du substantif, on observe que l'adjectif joue un rôle pour inverser ou atténuer la dimension axiologique du substantif, des adjectifs quasi-synonymes pouvant ne pas avoir le même fonctionnement. Ainsi remarque-t-on que si avec faible, l'atténuation peut se trouver transformée en une inversion, léger fonctionne plutôt comme atténuateur $^{8}$ d'amélioration, même si la valeur positive associée à amélioration y est encore affaiblie par la présence d'un adverbe de degré :

[10] Depuis la récession économique de 1982, on assiste à un affaiblissement de la capacité des syndicats de protéger les travailleurs et d'influencer l'orientation sociale. Le rapport de force qui leur était favorable dans les années 1960 et 1970 s'est renversé au profit du patronat. A certains égards, l'année 1988-1989 marque cependant une légère amélioration de la situation du syndicalisme quand on la compare aux cinq années antérieures. (L'année politique au Québec 1988-89, Le mouvement syndical, J. Rouillard, Université de Montréal, G)

[11] L'entourage de Guigou se félicite par ailleurs d'une légère amélioration de la situation du marché du travail pour les femmes (Le Monde, 21/6/02)

[12] La Commission estime qu'une très légère amélioration de ce rendement peut engendrer des gains importants en termes de rendement financier sur la quarantaine d'années que dure la vie professionnelle d'un travailleur, et réduire ainsi de manière significative le coût des retraites. (Libre circulation des capitaux, Bulletin UE 6-1997, Bruxelles-Luxembourg, G)

\footnotetext{
${ }^{8}$ L'analyse de S. Whittaker (2002 : 200, note 185) selon laquelle, en position d'épithète, léger serait spécifique à l'atténuation et faible à l'inversion ne nous semble juste que dans la seule mesure où le substantif est non marqué axiologiquement : par ex. une légère augmentation/une faible augmentation.
} 
Pour être inverseur, léger semble devoir être accompagné d'un focalisateur syntaxique. Ainsi, dans l'exemple [13], ne...que entraîne une focalisation spécialisée de l'adjectif (au sens de Nølke, 1994) et lui donne fonction d'inverseur :

[13] Elle pourrait retourner chez ses parents et reprendre ses études. Après tout cela ne faisait qu'un léger retard. On peut rattraper rapidement les cours qu'on a manqués. (B. Vian, L'Ecume des jours, 1947, cit. Lenepveu 2004)

Le focalisateur ne...que est indispensable ici pour que léger puisse inverser la valeur intrinsèquement négative de retard, alors qu'au vu des exemples [14] et [15] ci-dessous, faible peut soit atténuer, soit inverser la valeur d'un substantif marqué négativement comme risque, ce qu'il est possible de vérifier en utilisant les tests de substitution et de suppression. En effet, l'adjectif faible est inverseur dans [14] où il ne peut être ni remplacé par léger, ni supprimé si on veut maintenir la cohérence argumentative :

[14] Le 23 septembre 2005, le roquefort a bénéficié d'une autorisation de mise sur le marché de la part des autorités sanitaires australiennes. Le fromage français représente un faible risque pour la santé et la sécurité du consommateur, selon ces dernières. La nouvelle réjouit les professionnels du secteur (...) (franceagroalimentaire.com, Actualités du mois, 30/09/2005, G)

- ??Le fromage français représente un léger risque pour la santé et la sécurité du consommateur, selon ces dernières. La nouvelle réjouit les professionnels du secteur (...)

- ??Le fromage français représente un risque pour la santé et la sécurité du consommateur, selon ces dernières. La nouvelle réjouit les professionnels du secteur (...)

En revanche, dans l'exemple [15], faible est forcément atténuateur compte tenu du sens lexical du verbe et de l'orientation argumentative imposée par mais, l'adjectif pouvant d'ailleurs être supprimé ou remplacé sans difficulté par léger :

[15] Les grands progrès de la médecine ont apporté d'importantes améliorations de la sécurité des interventions, mais un faible risque persiste (Centre Fédéral d'Expertise des Soins de Santé, Communiqué de Presse, 21/10/04, G)

- mais un léger risque persiste

mais un risque persiste 
2. 2. Suite à cette analyse, nous nous sommes demandé si nous pouvions faire le même type d'observations quant au fonctionnement des adjectifs petit et maigre pris comme modificateurs déréalisants de consolation (une petite consolation/une maigre consolation). Autrement dit, l'hypothèse serait que, dans le discours, petite est atténuateur de consolation tandis que maigre est tantôt atténuateur, tantôt inverseur de ce même substantif. Il apparaît que maigre semble bien avoir les deux fonctionnements. Deux exemples :

[16] Les éleveurs qui ont perdu leur troupeau recevront 4000 dollars la tête ; une maigre consolation puisque chaque wapiti se vend le double en moyenne (Sciences et santé, Nouvelles, 19/12/00, G)

[17] Les résultats de dimanche n'ont fait que confirmer ses craintes. A Laval, François d'Aubert (UMP) a été réélu dès le premier tour de scrutin. Maigre consolation, le Parti Socialiste réalise dans cette ville un meilleur score qu'en 1997. (Le Monde, 12/06/02)

Dans le premier exemple [16], nous considérons que maigre inverse le trait positif associé à consolation, la perte de 4000 dollars étant difficile à percevoir comme une consolation. Dans l'exemple [17], nous pensons que maigre est atténuateur puisque l'argument donné peut très bien être perçu, cette fois-ci, comme une consolation, c'est-à-dire comme un événement désirable. Maigre pourrait même être remplacé par petite sans difficulté (Petite consolation, le parti Socialiste réalise dans cette ville un meilleur score qu'en 1997). On trouve d'ailleurs facilement des exemples attestés :

[18] Petite consolation pour les Européens, l'euro a bénéficié de l'effondrement des marchés boursiers américains en s'appréciant face au dollar. (Le Monde, 17/04/00)

La différence partielle de fonctionnement entre petite et maigre en position d'épithète antéposée n'est cependant pas toujours aussi nette que pour faible et léger. Tout d'abord, il n'est pas exclu de substituer petite à maigre quand maigre est inverseur, ce qui peut apparaître avec [16] déjà cité :

[16] Les éleveurs qui ont perdu leur troupeau recevront 4000 dollars la tête ; une maigre consolation puisque chaque wapiti se vend le double en moyenne

une petite consolation, puisque chaque wapiti se vend le double en moyenne 
Et surtout, on s'aperçoit, en multipliant les exemples, que l'opposition inverseur/atténuateur n'est pas toujours absolument manifeste. Ainsi, dans l'énoncé [19], nous pouvons difficilement dire si petit est atténuateur ou inverseur de succès, substantif également marqué positivement, dans la mesure où les deux interprétations sont compatibles avec l'orientation argumentative globale de l'énoncé :

[19] La problématique était : créer un logiciel de dessin facile à utiliser pour qui n'est pas informaticien (...). Ce fut Smartsketch. Petit succès commercial mais grande découverte, puisque les gens demandèrent à l'entreprise de convertir Smartsketch en logiciel d'animation. (Magazine Maison de l'image, novembre 2003, G)

De la même façon, dans [20], on peut difficilement dire si petit est inverseur ou atténuateur de avantage :

[20] On dira qu'une attitude de conciliation nous a valu quelques faveurs particulières. Petits avantages quand on songe à l'immense courant d'erreur et de mal qui envahit les âmes et les entraînent à l'apostasie ! Petits avantages qui nous enchaînent et qui nous empêchent de réagir contre nos adversaires. (Les évêques de France contre la laïcité en 1925, Lettre de l'Assemblée des Evêques de France publiée le 10 mars 1925, mise en ligne par B. Courcelle le 13/12/ 1998, G).

Le critère que pourrait constituer la présence du connecteur oppositif mais dans l'exemple [19] ne saurait être décisif car si mais révèle l'orientation argumentative de l'énoncé global, il ne signale pas nécessairement l'orientation axiologique du SN contenant le modificateur déréalisant : on sait que mais articule deux énoncés anti-orientés par rapport à une conclusion particulière ( $c f$. Ducrot, 1972), mais la conjonction n’implique pas de jugements appréciatifs d'orientations opposées.

Dès lors, pour savoir si un adjectif a la capacité de fonctionner comme inverseur, nous avons cherché à utiliser le fonctionnement d'un autre marqueur d'opposition moins lâche, et qui, lorsqu'il est combiné à mais signale, dans le cas d'une double attribution de propriétés comme ici, une opposition axiologique, il s'agit de l'adverbe néanmoins.

\section{Un critère décisif : mais néanmoins}


3. 1. Néanmoins est très souvent, dans les dictionnaires, rapproché de toutefois avec lequel il entretient des relations de synonymie partielle pour être opposé à pourtant et cependant ( $c f$. entre autres Lafaye, 1857 : 429-430). Le rapprochement est également fait chez Morel (1980) et (1996) pour qui les deux adverbes ont en commun de marquer, au sein des adverbes concessifs, une réserve de l'énonciateur sur les conclusions inférables de l'assertion précédente. En revanche, ils sont clairement distingués chez Blumenthal (1980), puis chez Nølke \& Gettrup (1984), néanmoins ne permettant jamais un glissement de sens vers la réfutation à la différence de toutefois. En d'autres termes, néanmoins construit, comme toutefois, une opposition, mais en signalant toujours le caractère non exclusif de l'opposition'

Nous nous sommes alors intéressée aux contextes qui privilégient néanmoins et en particulier à l'emploi très vivace de néanmoins dans les structures adjectivales coordonnées, lorsque l'adverbe est combiné avec mais ou avec et. Dans les deux cas, néanmoins construit une opposition tout en donnant les deux prédicats comme étant compatibles dans les circonstances présentes mais il est remarquable, à l'examen d'exemples attestés, qu'avec mais néanmoins, les adjectifs sont pris comme antonymes d'un point de vue axiologique. De plus, l'opposition n'est pas symétrique, mais orientée du négatif vers le positif. Précisément, le deuxième adjectif est intrinsèquement marqué positivement tandis que la construction marque négativement le premier adjectif, à moins que celui-ci ne soit déjà marqué intrinsèquement négativement ( $c f$. ex. [21]). Soit pour exemples :

[21] Question idiote mais néanmoins importante ( G)

[22] Petite annonce banale mais néanmoins vitale $(\mathrm{G})$

[23] Des instants de calmes brefs mais néanmoins marquants $(G)$

[24] la dimension tragique mais néanmoins glorieuse de ce mouvement $(\mathrm{G})$

[25] une vue partielle mais néanmoins encourageante de notre mission divine (G)

\footnotetext{
${ }^{9}$ C'est l'hypothèse que nous avons développée dans un article (à paraitre) consacré aux adverbes toutefois et néanmoins.
} 
[26] Un film ordinaire, certes, mais néanmoins intéressant et fascinant (G)

[27] Je vous remercie, monsieur le ministre, pour cette réponse un peu technocratique, mais néanmoins satisfaisante dans l'ensemble $(\mathrm{G})$

En revanche, les adjectifs peuvent avoir la même orientation axiologique quand néanmoins se combine avec et :

[28] une production télévisuelle diversifiée qui soit enrichissante et néanmoins divertissante (G)

[29] un serveur compact, hautes performances, fiable et néanmoins économique (G)

[30] Nikon EM dans la rubrique « téléchargements », pour tous ceux (celles) qui adorent ce charmant petit boîtier simple d'emploi et néanmoins très efficace $(\mathrm{G})$

[31] Douillettes et néanmoins spacieuses, ces suites avec vue sur le fairway ou la piscine disposent de vérandas indépendantes dans le salon et la chambre $(\mathrm{G})$

La mise en relation de deux propriétés apparemment contradictoires, possible avec mais néanmoins, nous paraît même exclue avec et néanmoins :

[32] Mail-zine à parution irrégulière mais néanmoins périodique / ??et néanmoins périodique (G)

Et inversement, la contradiction argumentative peut être suffisamment faible pour que mais soit inacceptable. A considérer l'énoncé [33], il n'est pas sûr, en effet, que dans l'esprit du locuteur, mais puisse être substitué à et :

[33] l'écoute des besoins et le souci de la réponse économiquement acceptable et néanmoins satisfaisant / ??mais néanmoins satisfaisant (G)

3. 2. C'est cette combinaison possible de mais avec néanmoins que nous voulons utiliser comme critère afin de repérer le fonctionnement des adjectifs modificateurs déréalisants, étant admis qu'en coordonnant deux syntagmes adjectivaux, mais néanmoins signale une opposition argumentative fondée sur une opposition axiologique.

On peut ainsi vérifier que petit, préférentiellement atténuateur en position d'épithète, est à même de fonctionner comme inverseur ${ }^{10}$ :

\footnotetext{
${ }^{10}$ D'autres structures concessives sont bien sûr à même de lever l'ambiguïté, tel un SPrep. introduit par malgré. Ainsi, dans l'exemple suivant, petit inverse nécessairement l'orientation de succès : Malgré un petit succès à l'étranger, le film rafle la plupart des prix de l'Australian Film Institute (Film de Culte, Portrait Mel Gibson, G).
} 
[34] Jusqu'en 1999, les deux sections encore en place [une section natation synchronisée et une section water-polo] ont continué leurs activités avec de petits, mais néanmoins encourageants succès (Montreux Natation Info, 2004, G)

Le critère choisi met aussi en évidence le rôle de la place occupée par l'adjectif, déjà souligné chez Ducrot (1995), puis chez S. Whittaker (2002). Ainsi, léger, que nous avons vu précédemment comme atténuateur d'amélioration en position d'épithète, va fonctionner comme inverseur lorsqu'il est en position d'attribut ${ }^{11}$ :

[35] Le marché français des mobiles gagne 500. 000 clients au deuxième trimestre. La hausse reste légère, mais néanmoins encourageante pour le secteur: le marché français des téléphones portables a progressé de $1,3 \%$ au deuxième trimestre, contre $0,8 \%$ le trimestre précédent. (Le $18 h$. com, Le quotidien de l'Expansion, $\left.\mathrm{n}^{\circ} 827,09 / 07 / 02, \mathrm{G}\right)$

Reste l'influence d'un adverbe de degré sur le fonctionnement de l'adjectif; les adjectifs eux-mêmes peuvent être modifiés et le choix du marqueur d'intensité portant sur l'adjectif contribue lui aussi à déterminer la valeur d'inverseur ou d'atténuateur de l'adjectif. On comparera à cet égard l'emploi des adverbes tout et bien, les deux adverbes ne jouant manifestement pas le même rôle dans la combinatoire des modificateurs.

\section{Quand tout et bien se combinent à un adjectif déréalisant}

Pour observer l'effet de tout et de bien sur le fonctionnement de l'adjectif déréalisant $^{12}$, nous choisissons d'opposer dans un premier temps une toute petite consolation à une bien maigre consolation. Il apparaît que tout ne fait que renforcer l'atténuation marquée par petite alors que bien, en renforçant la valeur dépréciative de maigre, favorise

\footnotetext{
${ }^{11}$ Dans un travail antérieur ( $c f$. Lenepveu, 2004 : §3), nous établissons un lien entre la focalisation de l'adjectif et l'orientation argumentative intrinsèque du substantif : pour être inverseur, l'adjectif doit pouvoir être focalisé, ce qui est le cas lorsqu'il est en position d'attribut puisqu'il est placé en frontière de phrase (cf. ex. [35]). Quand il est épithète, nous admettons l'hypothèse de Nølke (2001) selon laquelle le SN constitue un domaine de focalisation mineure et contient un focus, et nous reconnaissons que l'adjectif antéposé ne peut constituer à lui seul le focus simple, mais nous observons qu'il peut être soumis à une focalisation spécialisée qui n'est pas toujours marquée syntaxiquement par un élément focalisateur (seulement, ne...que, ...) et dans ce cas, fonctionner comme inverseur.

${ }^{12}$ Suivant les hypothèses de Ducrot (1998), les modificateurs agissent les uns sur les autres avant de s'appliquer aux prédicats.
} 
l'inversion du trait positif associé à consolation ${ }^{13}$. Nous utilisons ici comme critère l'emploi adverbial de juste, susceptible de contribuer à l'atténuation ${ }^{14}$ (juste une toute petite consolation) et incompatible avec les marqueurs qui peuvent induire une inversion (??juste une bien maigre consolation). Soit [36] où toute petite est atténuateur de consolation et compatible avec juste, puis [37] et [38] où bien maigre est inverseur de ce même substantif et incompatible avec juste:

[36] Ils ne gagnent plus, mais ils ne perdent pas non plus! C'est une toute petite consolation pour les Parisiens qui ont été tenus en échec par de solides Montpelliérains (0-0) (L'Equipe.fr, D1-8è journée, par Baptiste Blanchet, saison 2003-04, G)

juste une toute petite consolation

[37] La violence aujourd'hui est une sinistre réalité (...). Le fait que les citoyens « ordinaires » risquent moins de se faire assassiner que du temps de Restif de la Bretonne est une bien maigre consolation! Ils se demandent dans quels paradis idéologiques vivent les sociologues pour oser développer sans rire, pareilles calembredaines. (Le Monde, 21 juin 2002)

-?? juste une bien maigre consolation

[38] En se remplissant, une retenue d'eau anéantit l'écosystème de toute une vallée et prive ainsi l'homme des terres de culture et d'habitations. Les barrages bloquent les poissons migrateurs qui avaient l'habitude de remonter le courant. L'électricité produite en retour est finalement une bien maigre consolation, comparé au tribut que paye la nature. (Générations Futures.net, 20/12/04, G)

??juste une bien maigre consolation

Enfin, une toute petite consolation peut s'opposer à une bien petite consolation. Alors que toute maintient l'orientation du prédicat, la présence de bien inverse l'évaluation positive de

\footnotetext{
${ }^{13}$ Une bien maigre consolation s'oppose à une bien jolie fille où cette fois-ci bien renforce la valeur méliorative de jolie. Le fonctionnement de bien dépend manifestement du trait [ \pm désirable] associé à l'adjectif sur lequel il porte. Un exemple de Culioli (1988 : 169-170), et l'analyse qu'il en propose, nous paraît intéressant à cet égard. Soit: Elle est bien longue, cette planche. L'énoncé signifie que la longueur effective dépasse la longueur souhaitée mais il peut également indiquer que la longueur de la planche est la bonne longueur, c'est-à-dire la longueur souhaitée, dans le contexte suivant par exemple : Il me faudrait une planche un peu longue - Tiens, prends celle-là, elle est bien longue.

${ }^{14}$ Par exemple il y a juste un léger retard dans la transmission des dossiers. Nous adoptons l'analyse de Leeman (2004) selon laquelle juste, en tant qu'adverbe d'énonciation, sert à minimiser la portée de l'énoncé en neutralisant des inférences que l'interlocuteur pourrait tirer de cet énoncé.
} 
consolation; c'est bien qui confère cette fois-ci une valeur dépréciative à l'adjectif petite, et par là, favorise l'inversion du trait positif associé au substantif. Soit [39] :

[39] Produisant à la chaîne des milliers de chars Sherman, ils pouvaient facilement remplacer toutes les pertes encourues durant une bataille. Ceci, bien sûr, était une bien petite consolation pour les équipes de char Alliés se battant en Europe, et il n'était certes pas surprenant de voir les membres d'équipages recouvrir chaque recoin du blindage avec des sacs de sable. (Musée de la guerre.ca, 25/07/01, G)

Bien favorise l'inversion ; tout ne fait que renforcer l'atténuation. Ce que nous avons représenté sur l'échelle suivante :

[fig. 2]

$\begin{array}{ll}\text { une bien maigre/petite } & \text { une toute petite } \\ \text { consolation } & \text { consolation }\end{array}$

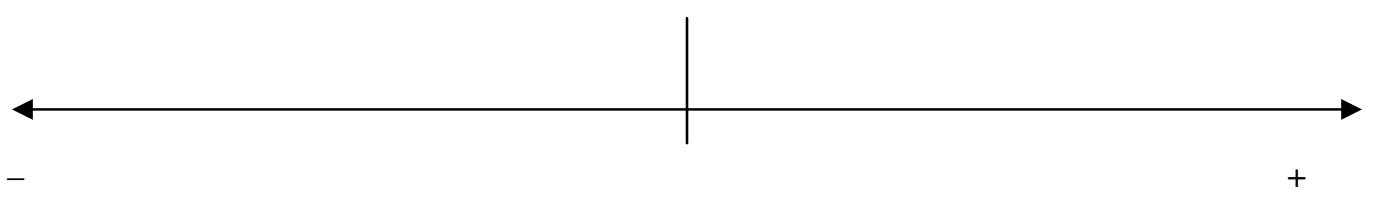

I

[40] et [41] illustrent aussi le fonctionnement de tout et de bien que nous cherchons à mettre en évidence, en opposant une toute petite amélioration à une bien faible amélioration et en utilisant le test que constitue pour nous l'emploi de l'adverbe juste:

[40] Une toute petite amélioration sur le plan financier. Continuez à rester prudent. Si vous vous sabotez ce début d'embellie, vous ne pourriez peut-être plus vous relever complètement, et vous auriez à le regretter amèrement. (Horoscope chinois du jour, Planète Québec, 05/08/05, G)

juste une toute petite amélioration

[41] Jusqu'à présent les prix ont fait preuve d'une remarquable inertie à la baisse, en dépit du caractère modeste de la reprise. Ainsi, selon Eurostat, l'inflation a certes reculé à 2,0\% en décembre, venant de 2,2\% en novembre, mais n'a fait que retrouver son niveau d'octobre. Sur l'ensemble de l'année, la hausse des prix aura atteint $2,1 \%$, une bien faible amélioration par rapport à 2002, où l'inflation s'était élevée à 2,2\%. (economic-research.bnpparibas.com, 07/08/05, G)

??juste une bien faible amélioration 
Afin de préciser cependant ce que nous entendons par un renforcement de l'atténuation, nous nous appuyons sur l'analyse que propose Bat-Zeev Shyldkrot (1995) du fonctionnement spatio-temporel de tout, et que nous étendons à la dimension argumentative. Selon l'auteur, tout est à même de localiser et circonscrire un point spécifié dans le temps et dans l'espace, point toujours défini comme une extrêmité initiale ou terminale. Alors qu'au fond de la salle peut signifier, nous citons Bat-Zeev Shyldkrot (1995: 81-82), «les trois ou quatre dernières rangées de la salle », tout au fond de la salle «se rapporte à un point extrêmement bien défini, qui constitue la limite même de la salle ». Si au début du cours peut signifier «dans le premier quart d'heure du cours », tout au début du cours veut dire «avant toute autre chose, tout de suite en entrant dans la classe ». De la même façon, nous dirons que le tout premier jour renvoie au point initial d'une série, donné comme un point extrême ${ }^{15}$ et nous poserons aussi l'hypothèse qu'une toute petite consolation ou une toute petite amélioration indiquent un point extrême dans le sens où toute petite désigne le plus faible des degrés orientés positivement, mais l'adverbe de degré bloque ici toute possibilité d'inversion, tandis que bien petite paraît imposer une réorientation de l'argumentation.

\section{Conclusion}

S'il a été montré que le discours construit une orientation axiologique qui peut se faire par l'inversion de la valeur axiologique de certaines entités linguistiques au moyen d'un adjectif déréalisant, on ne saurait considérer que celui-ci puisse être dit spécifique à l'inversion ou à l'atténuation. Dès lors, quand les tests classiques de suppression et de substitution nous paraissaient insuffisants, nous avons eu recours à la combinaison de mais avec le marqueur néanmoins, généralement considéré comme concessif, ou encore à l'emploi de juste comme adverbe d'énonciation, afin de lever toute ambiguïté interprétative et de mettre en évidence

\footnotetext{
${ }^{15}$ Dans une autre perspective, Franckel $(1989$ : 333) propose une analyse très proche d'exemples du type C'est
} 
les différents facteurs susceptibles d'influer sur la force évaluative du SN. Au total, quatre paramètres entrent en jeu : le SN lui-même, l'adjectif, la place de l'adjectif et le modificateur de l'adjectif, et ces quatre paramètres sont en relation avec les éléments du contexte pour déterminer la valeur axiologique du SN.

\section{BIBLIOGRAPHIE}

ANSCOMBRE J-Cl. (éd.), 1995, La théorie des topoï, Paris, Kimé.

BAT-ZEEV SHYLDKROT H., 1995, «Tout : Polysémie, grammaticalisation et sens prototypique », Langue Française, 107, p. 72-91.

BLINKENBERG A., 1933, L'ordre des mots en français moderne, 2, Kobenhavn, Levin \& Munksgaard.

BLUMENTHAL P., 1980, La syntaxe du message, Tübingen, Niemeyer.

CULIOLI A., 1988, « Autres commentaires sur bien », in BLANCHE-BENVENISTE C., CHERVEL

A. et GROSS M., Grammaire et histoire de la grammaire, Hommage à la mémoire de Jean Stefanini, Publications de l'Université de Provence, p. 169-180.

DUCROT O., 1972, Dire et ne pas dire, Paris, Hermann.

DUCROT O., 1980, Les échelles argumentatives, Paris, éd. de Minuit.

DUCROT O., 1995, « Les modificateurs déréalisants », Journal of pragmatics, 24, p.145-165.

DUCROT O., 1996, « Lexique et gradualité », in ALONSO E., BRUNA M. et MUNOS M., La Lingüistica francesa : gramatica, historia, epistemologia, Séville, p.191-205.

DUCROT O., 1998, «Quand peu et un peu semblent co-orientés », in LEEMAN D., BOONE A. et alii., Du percevoir au dire, Hommage à André Joly, L'Harmattan, p. 351-373.

FRANCKEL J-J., 1989, Etude de quelques marqueurs aspectuels du français , Genève-Paris, Droz. GALATANU O., 2002, « La dimension axiologique de l'argumentation », in CAREL M., Les facettes du dire. Hommage à Oswald Ducrot, Paris, Kimé, p. 93-107.

son tout premier disque ou son tout premier essai : «Tout premier introduit une orientation rétrospective dans le 
GETTRUP H. \& NØLKE H., 1984, «Stratégies concessives : une étude de six adverbes français », Revue Romane, 19-1, p. 3-47.

GOES J., 1999, L'adjectif. Entre nom et verbe, Paris-Bruxelles, Duculot.

GOSSELIN L., 2005, Temporalité et modalité, Duculot-De Boeck, Bruxelles.

HADERMANN P., PIERRARD M., VAN RAEMDONCK D. (éds), 2007, « La scalarité : autant de moyens d'expression, autant d'effets de sens », Travaux de linguistique, 54.

KENNEDY C. \& McNALLY L., 1999, «From Event Structure to Scale structure: Degree Modification in Deverbal Adjectives », in MATTHEWS T. et STROLOVITCH D., Proceedings of SALT, 9, Ithaca, CLC Publications, p. 163-180.

KENNEDY C., 1999, Projecting the adjective. The syntax and semantics of gradability and comparison, New-York \& London, Garland.

LAFAYE B., 1857, Dictionnaire des synonymes de la langue française, librairie Hachette.

LEEMAN D., 2004, «L'emploi de juste comme adverbe d'énonciation », Langue Française, 142, p. $17-30$.

LENEPVEU V., 2002, « Adjectifs et adverbes : une corrélation syntactico-sémantique », Le français moderne, 70,1, p. 45-70.

LENEPVEU V., 2004, « La valeur intensive de l'adjectif antéposé », Travaux linguistiques du CERLICO, 17, Presses Universitaires de Rennes, p. 51-66.

LENEPVEU V ., (à par.), «Toutefois et néanmoins, une synonymie partielle », Syntaxe et Sémantique, 8, Presses Universitaires de Caen.

MARTIN R., 1986, «Le vague et la sémantique de l'adjectif. Réflexions sur l'adjectif antéposé », Quaderni di Semantica, 7, 2, p. 246-243.

MILNER J-Cl., 1967, « Esquisse à propos d'une classe limitée d'adjectifs en français moderne », MIT, Quaterly Progress Report, 84, Research Laboratory of Electronic, p. 275-285.

MOREL M-A., 1980, Etudes sur les moyens grammaticaux et lexicaux propres à exprimer une concession en français contemporain, Lille, Atelier National de Reproduction des Thèses.

MOREL M-A., 1996, La concession en français, Paris-Gap, Ophrys.

temps. Tout marque que dans cette orientation, il n'existe pas d'au-delà de ce premier ». 
NØJGAARD M., 1992-93-95, Les adverbes français. Essai de description fonctionnelle, 3 vols., Historisk-filosofiske Meddelelser, 66, Copenhague, Munksgaard.

NØLKE H., 1994, Linguistique modulaire : de la forme au sens, Louvain-Paris, Peeters.

NØLKE H., 2001, Le regard du locuteur 2, Paris, Kimé.

RACCAH P-Y., 2005, « Une description de l'excessivité en sémantique des points de vue », Travaux linguistiques du CERLICO, 18, Presses Universitaires de Rennes, p. 171-190.

RIEGEL M., 2005, «Une ancienne chapelle, un pur mensonge, un vague diplôme: ou quand un simple adjectif modalise le rapport de la désignation nominale », Cahiers de lexicologie, 86, p. 105129.

RIVARA R., 1993, «Adjectifs et structures sémantiques scalaires », L'information grammaticale, 58, p. $40-46$.

ROMERO C., 2001, L'Intensité en français contemporain : analyse sémantique et pragmatique, thèse de doctorat en Sciences du Langage, Université de Paris 8.

WHITTAKER S., 2002: La notion de gradation. Applications aux adjectifs, Publications Universitaires Européennes, Peter Lang.

WILMET M., 1980: «Antéposition et postposition de l'épithète qualificative en français contemporain », Travaux de linguistique, 7, 179-201. 